\title{
A REDUCTION IN NUMBER OF THE POSTULATES FOR C. I. LEWIS' SYSTEM OF STRICT IMPLICATION*
}

BY J. C. C. McKINSEY

C. I. Lewis assumes the following undefined ideas:

1. Propositions: $p, q, r$, etc.

2. Negation: $\sim p$.

3. Logical product: $p q$, or $p \cdot q$.

4. Self-consistency or possibility: $\diamond p$.

5. Logical equivalence: $p=q$.

In terms of the above ideas, he defines implication and equivalence as follows:

$$
\begin{aligned}
& p<q \cdot=: \sim \diamond(p \sim q), \\
& p=q \cdot=: p<q \cdot q<p .
\end{aligned}
$$

Lewis asserts among others the following symbolic postulates:

$$
p q \cdot<\cdot q p
$$

$$
p q \cdot<\cdot p
$$

$$
p \cdot<\cdot p p,
$$

11.5

$$
p \cdot<\cdot \sim \sim p
$$

11.6

$$
p<q \cdot q<r:<\cdot p<r .
$$

He allows the following operations:

Substitution. (a) Either of two equivalent expressions may be substituted for the other. (b) Any proposition, or any expression which has meaning in terms of the undefined ideas, may be substituted for $p$, or $q$, or $r$, etc., in any assumption or established theorem.

Adjunction. If $p$ has been asserted and $q$ has been asserted, then $p q$ may be asserted.

Inference. If $p$ has been asserted and $p<q$ has been asserted, then $q$ may be asserted.

* Symbolic Logic, by Lewis and Langford, pp. 123-126. 
I show as follows how postulate 11.5 may be deduced from 11.1, 11.2, 11.3, and 11.6.

Theorem 1. $p<p$.

$$
\begin{array}{ll}
{[11.3]} & p<p p \\
{\left[11.2, \frac{p}{q}\right]} & p p<p \\
{\left[11.6, \frac{p p}{q}, \frac{p}{r}\right]} & p<p p \cdot p p<p:<\cdot p<p \\
{[(1),(2),(3)]} & p<p,
\end{array}
$$

which was to be proved.

Theorem 2. $p q=q p$.

$$
\begin{array}{ll}
{\left[11.1, \frac{p}{q}, \frac{q}{p}\right]} & q p<p q \\
{[11.1]} & p q<q p \\
{[(1),(2), 11.03]} & p q=q p,
\end{array}
$$

which was to be proved.

Theorem 3. $\sim \sim p<p$.

$$
\begin{array}{ll}
{\left[\text { Th. } 1, \frac{\sim p}{p}\right]} & \sim p<\sim p \\
{[(1), 11.02]} & \sim \vee(\sim p \sim \sim p) \\
{\left[\text { Th. } 2, \frac{\sim p}{p}, \frac{\sim \sim p}{q}\right]} & \sim p \sim \sim p=\sim \sim p \sim p \\
{[(2),(3)]} & \sim \vee(\sim \sim p \sim p) \\
{[(4), 11.02]} & \sim \sim p<p,
\end{array}
$$

which was to be proved.

TheOREM $4 . p<\sim \sim p$.

[Th. $\left.3, \frac{\sim \sim p}{p}\right] \sim \sim \sim \sim p<\sim \sim p$

[Th. 3,]

$$
\sim \sim p<p
$$


$[(1),(2), 11.6] \quad \sim \sim \sim \sim p<p$

[(3), 11.02] $\sim \wedge(\sim \sim \sim \sim p \sim p)$

$\left[\right.$ Th. $\left.2, \frac{\sim \sim \sim \sim p}{p}, \frac{\sim p}{q}\right]$

$\sim \sim \sim \sim p \sim p=\sim p \sim \sim \sim \sim p$

$[(4),(5)]$

$\sim \diamond(\sim p \sim \sim \sim p)$

$[(6), 11.02]$

$\sim p<\sim \sim \sim p$

[Th. $\left.3, \frac{\sim p}{p}\right]$

$\sim \sim p<\sim p$

$[(7),(8), 11.03] \quad \sim p=\sim \sim \sim p$

[Th. 1, 11.02]

$\sim \diamond(p \sim p)$

[(10), (9)]

$\sim \diamond(p \sim \sim \sim p)$

[(11), 11.02]

$p<\sim \sim p$

which was to be proved.

Theorem 4 is Lewis' postulate 11.5.

New York University

\section{NOTES ON THE AIRY STRESS FUNCTION}

\section{BY J. H. A. BRAHTZ}

1. The Expressions for the Components of Stress. Problems in plane stress and plane strain are usually best treated by use of the Airy stress function even when body forces exist. It has been customary to express the stresses as follows:

$$
\text { (1) } \sigma_{x}=\frac{\partial^{2} A}{\partial y^{2}}-C_{1} x, \quad \sigma_{y}=\frac{\partial^{2} A}{\partial x^{2}}-C_{2} y, \quad \tau_{x y}=-\frac{\partial^{2} A}{\partial x \partial y},
$$

where $C_{1}$ and $C_{2}$ are components of the uniform body force $C$ per unit volume acting at an angle $\beta$ with the $x$-axis. Hence

$$
C_{1}=C \cos \beta, \quad C_{2}=C \sin \beta .
$$

It will be found that an arbitrary function $A(x, y)$ will satisfy the equations of equilibrium for a rectangular element $d x d y$ : 